\title{
Does the Level of Chlamydia Serology Titre Correlate to the Degree of Tubal Disease at Laparoscopy? A Retrospective Controlled Study
}

\author{
Abdu $\mathrm{N}^{1}$, Bokhari $\mathrm{M}^{2}$ and Al-Inizi $\mathrm{S}^{* 1}$ \\ ${ }^{1}$ Department of Obstetrics and Gynaecology, South Tyneside Hospital, South Shields, UK \\ ${ }^{2}$ Research Department, Queen Elizabeth Hospital, Gateshead, UK
}

*Corresponding author: Al-Inizi S, Consultant Obstetrician and Gynaecologist, South Tyneside Hospital, Harton Lane, NE340PL, South Shields, UK, Fax: +1912024019, Tel: +441914041000, E-mail: shamma.alinizi@stft.nhs.uk

Citation: Abdu N, Bokhari M, Al-Inizi S (2017) Does the Level of Chlamydia Serology Titre Correlate to the Degree of Tubal Disease at Laparoscopy? A Retrospective Controlled Study. J Gynecol Res 3(2):203. doi: 10.15744/2454-3284.3.203

Received Date: October 24, 2017 Accepted Date: December 27, 2017 Published Date: December 29, 2017

\begin{abstract}
Objectives: In order to detect if there is any correlation between the level of total and specific Chlamydia serology titre and the degree of tubal disease at laparoscopy in sub fertile women and to compare the results with a control group.

Study Design: A retrospective controlled clinical study was conducted in a hospital setting (both inpatient and outpatient clinic). A total of hundred and twenty two sub fertile women who underwent laparoscopy/tubal surgery in the period between January 2011-January 2014 were included. This included 40 women with positive Chlamydia Trachomatis specific titre (group 1), 40 with positive Chlamydia serology total titre only (group 2) and 42 women with negative Chlamydia serology titre (control group).

Chlamydia serology titre was measured by ELISA. The differentiated Chlamydia specific titres were measured using Microscopic Immunofluorescence Assay (MIF) to determine the immunity status. The Chlamydia Trachomatis IgG titres were classified for the purpose of the study into normal $(<1 / 16)$, Mild (1/16), Moderate $(1 / 32)$, and severe $(>=1 / 64)$.

Laparoscopic grading of tubal disease was performed into grades 1-3 according to the severity of the tubal damage which was compared to the Chlamydia titre. Pearson`s correlation test was used as a descriptive measure.

Result: There is a subjective correlation between Chlamydia serology titre and the degree of tubal disease at laparoscopy. It was subjectively evident that the higher the Chlamydia serology titre, the more severe was the tubal disease at laparoscopy, however; this was not statistically significant. There was subjective evidence that Chlamydia Trachomatis specific titre (group 1) was more indicative of the degree of tubal disease than the high total Chlamydia titre (group 2). Grade 2 tubal diseases were the most common grade identified in the study and most women had secondary rather than primary sub-fertility.

Conclusion: Our clinical data from sub fertile women with positive Chlamydia serology indicate a subjective correlation with the degree of tubal disease especially in those with high Trachomatis specific titre, which was not statistically significant. Counselling sub fertile women with positive Chlamydia serology regarding this correlation remains an essential part of the subfertility consultation.

Keywords: Chlamydia Serology; Tubal Disease; Subfertility
\end{abstract}

\section{Introduction}

Sub fertility is a condition of the reproductive system defined by the failure to achieve a clinical pregnancy after 12 months or more of regular unprotected sexual intercourse [1]. Global sub fertility prevalence rates are difficult to determine. It is not possible to synthesize infertility prevalence data in the published literature because of the incomparable definitions used [2] Demographic and Health Surveys by the World Health Organization (WHO) revealed that one in every four couples in developing countries had been found to be affected by infertility and one in seven couples in the UK $[1,3]$.

The burden remains high. A WHO study published in 2012, which applied a consistent algorithm to demographic and reproductive surveys available from developed and developing countries, and estimated infertility prevalence and trends with the overall burden of infertility in women from 190 countries has remained similar in estimated levels and trends from 1990 to 2010 [3].

Chlamydia is one of the most common sexually transmitted infections in the UK. It's transmitted during unprotected intercourse 
by the bacterium Chlamydia Trachomatis. For the year 2011, 86,698 men and 126,129 women were diagnosed in the UK. There was an overall $2 \%$ reduction in diagnoses since the previous year. This fall may have been a result of the national screening programme [4].

Chlamydia is a genus of bacteria that are obligate intracellular parasites. There are three subspecies that affect human; C. Trachomatis, C. pneumoniae and C. psittaci [5].

C. Trachomatis is the one responsible for the sexually transmitted disease. Most commonly, Chlamydial infections do not cause symptoms. However, for men a burning sensation when urinating is often probable. For women, odour and itching are possible symptoms. Chlamydial infection can spread to the uterus or fallopian tubes and cause pelvic inflammatory disease (PID). PID can damage the fallopian tubes and uterus and cause chronic pelvic pain, tubal disease - sub fertility, and ectopic pregnancy. Women who had Chlamydia also are at much greater risk for becoming infected with HIV [1].

Although the prevalence of C. Trachomatis among sub-fertile women in the UK is only 1.9\%, uterine instrumentation carried out routinely as part of the infertility investigations may reactivate or introduce upper tract dissemination of endo-cervical Chlamydial infection, resulting in iatrogenic pelvic inflammatory disease [1]. Previous studies suggested that Chlamydial infection, as evidenced by positive antibody titre, is associated with a significantly high incidence of tubal infertility [6].

Chlamydia Trachomatis IgG antibody testing has been recommended to be the first screening test for tubal factor sub fertility because of its high specificity and fertility- enhancing effect [7]. The Chlamydia titre can give additional information.

The correlation between the total and the Trachomatis specific Chlamydia titre and the degree of tubal disease was not studied before. In this study we have looked at the total Chlamydia titre with and without raised Trachomatis specific titre and the degree of tubal disease at laparoscopy. We compared these results with a control group of sub fertile women who had negative Chlamydia serology, some of whom were found to have tubal disease at laparoscopy due to other causes.

\section{Materials and Methods}

This was a retrospective descriptive controlled study of patients from the sub-fertility clinic at South Tyneside Hospital during the period from January 2011 to January 2014 who was found to have positive Chlamydia serology and have undergone laparoscopic surgery. A control group of women with subfertility and negative Chlamydia serology who underwent laparoscopy was included. Chlamydia serology titre was measured to determine the immunity status as part of the routine investigations for all women with sub-fertility. Not all women who were found to be positive for Chlamydia gave history of previous Chlamydia infection. Microscopic immunofluorescence assay (MIF) was used to measure the Chlamydia specific serology titres and ELISA was used to measure the total Chlamydia serology titre. MIF is considered the gold standard of Chlamydia species specific serology. It is highly sensitive (80\%) with high degree of sero-diagnostic specificity (91\%) in certain Chlamydia infections. The results don't change with time unless re infection happens. However, cross reactivity with $\mathrm{C}$ Pneumonia antibodies was reported with some of the peptide based ELISA tests [8].

The Chlamydia Trachomatis specific IgG titre levels were classified for the purpose of the study to Normal $(<1 / 16)$, Mild $(1 / 16)$, Moderate $(1 / 32)$, and severe $(=>1 / 64)$.

Below is an example of a normal Chlamydia Serology laboratory result showing the total (IgG), sub type of Chlamydia and the IgA titre which indicates current Chlamydial infection (Table 1).

\begin{tabular}{|c|c|}
\hline Total Chlamydia IgG & $<30 \mathrm{EIU}$ \\
\hline Chlamydia IgA & Negative \\
\hline CHLAMYDIA ANTIBODIES & not detected \\
\hline $\begin{array}{c}\text { CHLAMYDIA MIF (specific } \\
\text { titre) }\end{array}$ & \\
\hline C. trachomatis & $\operatorname{IgG}<1 / 16, \operatorname{IgA}<1 / 8, \operatorname{IgM}<1 / 8$ \\
\hline C. pneumoniae & $\operatorname{IgG}<1 / 16, \operatorname{IgA}<1 / 8, \operatorname{IgM}<1 / 8$ \\
\hline C. psittaci & $\operatorname{IgG}<1 / 16, \operatorname{IgA}<1 / 8, \operatorname{IgM}<1 / 8$ \\
\hline
\end{tabular}

EIU: enzyme immunounits; MIF: Micro-Immunofluorescence test

Table 1: Chlamydia Serology laboratory results indicating total (IgG) and subtypes of Chlamydia

Women were subdivided into three groups according to the level of Chlamydia titre:

Group 1: A group with high Chlamydia Trachomatis (specific) IgG titre (>1/16)

Group 2: A group with high total Chlamydia titre but normal Trachomatis specific titre ( $>30 \mathrm{EIU})$

Group 3: A Control group with negative Chlamydia titre (<30EIU)

Laparoscopic findings were compared to the Chlamydia serology titre, and the degree of tubal disease. Grading of tubal disease happened in theatre by the same operator who was aware of the Chlamyda serology before the operation. This was graded according to the following classification [9]: 
GRADE $1 \quad$ Both tubes patent, with minimal unilateral or bilateral tubo-ovarian adhesions

GRADE 2 Unilateral tubal block with or without hydrosalpinx with patent other tube with or without tubo-ovarian adhesions

GRADE 3 Bilateral tubal block with bilateral hydrosalpinx

This classification system reliably distinguishes infertile patients with tubal disease into favourable and unfavourable groups which is useful as subsequent management would depend on this assessment, especially as the classification is able to define which group of patients would benefit most from interventions such as surgery. Women with tubal disease are categorized into prognostic groups using this simple classification system based on severity [9].

Pearson's correlation test was used as a descriptive measure for statistical analysis.

\section{Results}

A total of 122 patients were included in the study. They were classified into three groups according to their total and specific Chlamydia titres as follows:

Secondary sub fertility was more observed in group 1 (62\%) rather than primary sub fertility, in comparison to groups 2 \& 3 which had equal primary and secondary subfertility.

Tubal disease was more commonly observed in group 1 (69.3\%) compared to groups 2 \& 3 (30\% \& 38.1\% respectively). Group 1 had the highest number of grades $2 \& 3$ tubal disease (Table 2).

\begin{tabular}{|c|c|c|}
\hline GROUP 1 & Chlamydia Trachomatis titre $>1 / 16$ & 40 patients \\
\hline GROUP 2 & $\begin{array}{c}\text { Total Chlamydia titre }>30 \text { EIU with } \\
\text { normal Trachomatis titre }\end{array}$ & 40 patients \\
\hline $\begin{array}{c}\text { GROUP 3 } \\
\text { (control) }\end{array}$ & Negative Chlamydia Titre $<30 \mathrm{EIU}$ & 42 patients \\
\hline
\end{tabular}

Table 2: Classification of the three groups according to the Chlamydia serology titre

The study showed that the degree of tubal disease subjectively correlates positively with the level of Chlamydia serology titre in group 1, however; this was not statistically significant (P0.346), (Figure 1). This could be due to the broad individual differences in response to Chlamydia infection or possibly due to the small sample size used in the study.

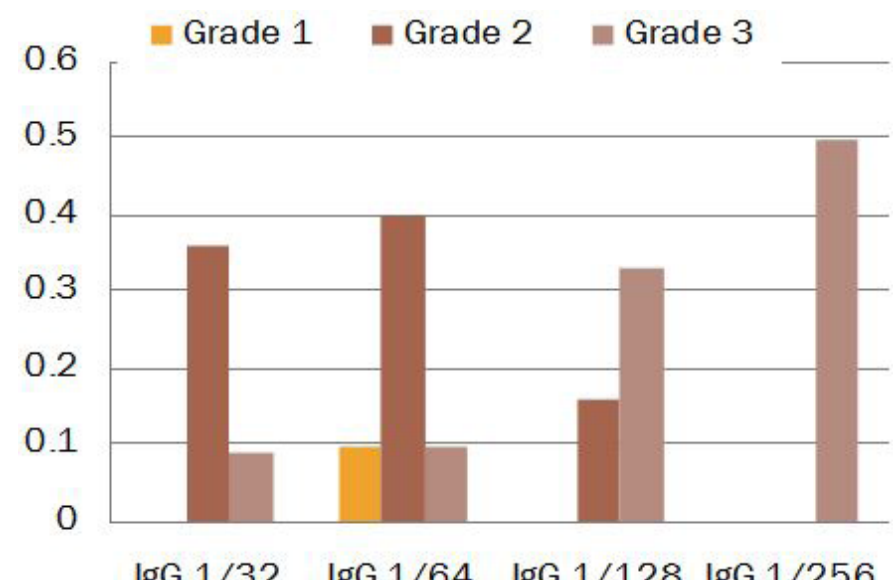

$\lg 1 / 32 \operatorname{lgG} 1 / 64 \lg 1 / 128 \lg 1 / 256$

Figure 1: Severity of tubal disease according to the level of Chlamydia Trachomatis titre in group 1

It was found that tubal disease was more pronounced when the Chlamydia Trachomatis titre was more than 1/64.

The most common grade of tubal disease associated with previous Chlamydia infection was found in grades $2 \& 3$. It was found in 24/40 and 10/40 of the women in groups 1 and 2, respectively (Table 3). Grade 1 tubal disease was the least commonly seen in women with previous Chlamydia infection.

Grade 3 tubal disease was more commonly found in women with higher Chlamydia Trachomatis serology titre (Table 3 and Figure 1). Grade3 tubal disease was seen in 30\% of women with high Chlamydia Trachomatis titre (group 1), 10\% of women with high total Chlamydia titre (group 2), and only found in $4.7 \%$ of women with negative Chlamydia titre in group 3 (Table 3 ). The tubal disease found in group 3 was mainly due to pelvic endometriosis. Women with high total Chlamydia titre and negative Trachomatis specific titre (group 2) can also develop tubal disease to a lesser extent which was found not to be statistically significant (P 0.67). The commonest surgical intervention performed during laparoscopy was division of tubo ovarian adhesions/ tubal surgery and HELICA coagulation of endometriosis. Occasionally, division of large hydrosalpinx \& salpingectomy were performed to improve 
implantation rate and to prepare for IVF when required [10].

\begin{tabular}{|c|c|c|c|}
\hline $\begin{array}{c}\text { Severity (grading) } \\
\text { of tubal disease }\end{array}$ & Group 1 (\%) & Group 2 (\%) & Group 3 (\%) \\
\hline 0 & 30.8 & 70.0 & 61.9 \\
\hline 1 & 7.7 & 2.5 & 9.5 \\
\hline 2 & 30.8 & 15.00 & 23.8 \\
\hline 3 & 30.8 & 12.5 & 4.8 \\
\hline Total & 100 & 100 & 100 \\
\hline
\end{tabular}

Table 3: Illustrating severity of tubal disease Vs Grouping. This is expressed as percentages

\section{Discussion}

Chlamydia is one of the commonest sexually transmitted infections. The infection could cause mild symptoms if any, but its long term squeal mainly on fertility could be very serious. Chlamydia Trachomatis is the main causative organism.

Before undergoing uterine instrumentation such as hysterosalpingogram and laparoscopy/dye test, sub fertile women should be offered screening for Chlamydia Trachomatis using an appropriately sensitive technique [1].

Chlamydia Trachomatis IgG antibody testing has been recommended to be the first screening test for tubal factor sub fertility because of its high specificity and fertility- enhancing effect [11]. It was recognised in this study that the Chlamydia titre can give additional information.

It has been previously reported that Chlamydia Trachomatis serology levels are quantitatively related to the severity of tubal damage in infertile women. Wide variations in the severity of lesions observed in relation to serology levels were suggestive of broad individual differences in response to Chlamydial infection [11].

The relationship between the total and specific Chlamydia serology titre and the degree of tubal disease at laparoscopy was not reported before. We studied this correlation retrospectively and compared the results to a control group of sub fertile women with negative Chlamydia serology who had laparoscopy during their sub fertility investigations.

We subjectively found that women with positive Chlamydia Trachomatis (group 1) were mostly diagnosed with tubal disease which was mostly found in grades $2 \& 3$, however; this was not found to be statistically significant. We think that this could be related to the broad individual differences in response to Chlamydia infection which has been reported before (11)or possibly due to the small sample size used in the study.

We also subjectively found that the more severe the Chlamydial infection (indicated by higher Chlamydial serology titre); the more severe is the tubal disease which was again found not to be statistically significant. Women with only positive total Chlamydia titre (group 2) can still have tubal disease but to a lesser extent which was also found not to be statistically significant. Women with negative Chlamydia serology (group 3) can still have tubal disease caused by other factors such as endometriosis. As the correlation between the Chlamydia serology titre and tubal disease was found not to be statistically significant; its clinical value remains uncertain; however; its observational correlation can be important especially when tubal disease is found on hysterosalpingogram (e.g tubal block with hydrosalpinx) indicating the need for possible laparoscopy and tubal surgery. On the other hand, women with raised Chlamydia serology titre and normal tubes on hysterosalpingogram might not require a laparoscopy during their fertility journey.

\section{Conclusion}

Chlamydia serology titre (both total and specific) remains an important part of the subfertility investigations. There is a subjective observational correlation between total and specific Chlamydia serology titre and the degree of tubal disease at laparoscopy which was found not to be statistically significant. This is a preliminary study and a larger study would possibly have clearer results regarding this correlation. Counselling sub fertile women with raised Chlamydia serology titres regarding this subjective correlation with the degree of tubal disease considering individual unpredicted variations in response to the Chlamydia infection is an important part of the sub fertility consultation.

\section{Acknowledgement}

Dr. Mira Bapir, Dr. Jennifer Hoh, Dr. Adriane Harrop who participated in the data collection of this study.

\section{References}

1. National Institute of Clinical Excellence (2013) Fertility problems: assessment and treatment, Guideline no. 156. NICE guidelines [CG156], UK.

2. Gurunath S, Pandian Z, Anderson RA, Bhattacharya S (2011) Defining infertility a systematic review of prevalence studies. Hum Reprod Update 17: 575-88.

3. Mascarenhas MN, Flaxman SR, Boerma T, Vanderpoel S, Stevens GA (2012) National, Regional and Global Trends in Infertility Prevalence Since 1990: A Systematic Analysis of 277 Health Surveys. PLoS Med 9: e1001356. 
4. Table 13: Number and rates of selected STI diagnoses in the UK, 2008 - 2011; Public Health England, UK.

5. Ryan KJ, Ray CG (2004) Sherris Medical Microbiology (4 $4^{\text {th }}$ Edn) McGraw Hill, USA.

6. Kelver ME, Nagamani M (1989) Chlamydia serology in women with tubal infertility. Int J Fertil 34: 42-5.

7. den Hartog JE, Lardenoije CM, Severens JL, Land JA, Evers JL, et al. (2008) Screening strategies for tubal factor subfertility. Hum Reprod 23: 1840-8.

8. Clad A, Freidank H, Plünnecke J, Jung B, Petersen EE (1994) Chlamydia trachomatis species specific serology: ImmunoComb chlamydia bivalent versus microiimunoflurescence (MIF). Infection 22: 165-73.

9. Akande VA. Tubal Disease: towards a classification. Reprod Biomed Online 15: 369-75.

10. Déchaud H, Daurès JP, Arnal F, Humeau C, Hédon B (1998) Does previous salpingectomy improve implantation and pregnancy rates in patients with severe tubal factor infertility who are undergoing in vitro fertilization? A pilot prospective randomised study. Fertility Sterility 69: 1020-5.

11. Elhakim EA, Gordon UD, Akande VA (2010) The relationship between serum Chlamydia antibody levels and severity of disease in infertile women with tubal damage. Arch Gynecol Obstet 281: 727-33.

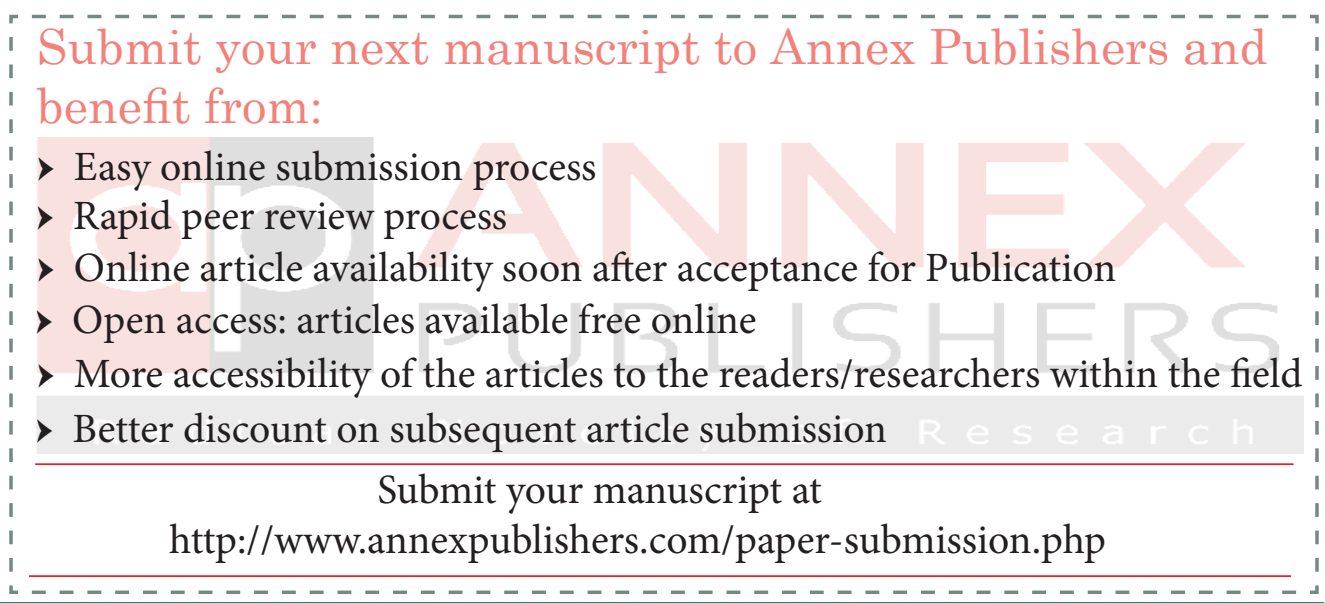

